\title{
NONCONVEX DUALITY AND SEMICONTINUOUS PROXIMAL SOLUTIONS OF HJB EQUATION IN OPTIMAL CONTROL*
}

\author{
Mustapha Serhani $^{1}$ AND NADiA RAÏSsI ${ }^{2}$
}

\begin{abstract}
In this work, we study an optimal control problem dealing with differential inclusion. Without requiring Lipschitz condition of the set valued map, it is very hard to look for a solution of the control problem. Our aim is to find estimations of the minimal value, $(\alpha)$, of the cost function of the control problem. For this, we construct an intermediary dual problem leading to a weak duality result, and then, thanks to additional assumptions of monotonicity of proximal subdifferential, we give a more precise estimation of $(\alpha)$. On the other hand, when the set valued map fulfills the Lipshitz condition, we prove that the lower semicontinuous (1.s.c.) proximal supersolutions of the Hamilton-Jacobi-Bellman (HJB) equation combined with the estimation of $(\alpha)$, lead to a sufficient condition of optimality for a suspected trajectory. Furthermore, we establish a strong duality between this optimal control problem and a dual problem involving upper hull of l.s.c. proximal supersolutions of the HJB equation (respectively with contingent supersolutions). Finally this strong duality gives rise to necessary and sufficient conditions of optimality.
\end{abstract}

Keywords. Optimal control, duality, HJB equation, proximal supersolution, proximal subdifferential.

Mathematics Subject Classification. 49J24, 49K24, 49N15, 49L99, 49J52.

\footnotetext{
Received January 30, 2006. Accepted February 2, 2009.

* This work was partially financed by the PROTARS P2T01/09

1 Département d'économie, FSJES, Université My Ismail, B.P. 3102, Toulal, Meknès, Maroc; mserhani@hotmail.com

2 EIMA, Département de Mathématiques et d'Informatique, Faculté des Sciences, Université Ibn Tofail, B.P. 133, Kénitra, Maroc; n.raissi@lycos.com 


\section{INTRODUCTION}

In this work, we study a Mayer optimal control problem dealing with differential inclusion. Many works are interested by the same problem, giving rise to characterization of optimal solutions on terms of solutions of Hamilton-Jacobi-Bellman Equation (HJB). These results are obtained under a Lipschitz condition of the set valued map (or the single valued map if we deal with differential equation), see for instance $[12,15,21-23,33]$. But it is well known that there are several situations where Lipschitz condition is not fulfilled. One of them is the differential inclusion theory. In this framework, the Lipschitz condition not appear to be a standing assumption under which we look for solution, equilibrium, stability and asymptotic behavior of trajectories. The standing assumptions are the so called Peano assumptions, that is, the set valued map of differential inclusion is upper semicontinuous with nonempty, compact, convex values and satisfies the linear growth condition (see [4], Chap. 10, and [1,2]). On the other hand, for many problems arising from different areas and formulating in terms of the following optimal control problem:

$$
\begin{gathered}
\left(P_{c}\right) \quad \inf _{x} J(x):=g(x(T)) \\
\dot{x}(t)=f(t, x(t), u(t)) \text { a.e. } t \in[0, T] \\
u(t) \in U \text { a.e. } t \in[0, T], \quad x(0)=x_{0} .
\end{gathered}
$$

Equation (0.1) may take several sophisticated forms as closed loop control $\dot{x}(t)=$ $f(t, x(t), u(t, x(t)))$ or $\dot{x}(t)=A(x(t)) \frac{\mathrm{d}}{\mathrm{d} t}(B(x(t))+C(x(t))$ where the velocity depends not only upon the state $x$ but also on variation of observations $B(x)$ or potential $\dot{x}(t)=-\nabla V(x(t)), \ldots$ In such situations, often the control $u(t, x)$ is not Lipschitz, this is the case of bioeconomic models where we deal with capital and investment (control) with infinite value (this means that the control is discontinuous and hence the Lipschitz condition is not fullfieled, such phenomenas are economically viewed as instantaneous jump of capacity, see [11]). Similarly the observation or potential are often not continuous and instead $\nabla V(x(t))$ we invoke the subdifferential $\partial V(x(t))$ (see [2], introduction). Finally, when we reduce the problem $P_{c}$ to a differential inclusion problem, by choosing the parameterized set valued map $F$ associated with $f$ and defined by $F(t, x):=\{f(t, x, u): u \in U\}$. The continuity of $f$ (the most smoothness property does generally found) is not enough to guarantee that $F$ is Lipschitz. Indeed, from the celebrate Filippov lemma, $F$ is only upper semicontinuous (see Prop. 15, Chap. 3, [3], [2,4] and [15], Ex. 1.3, Chap. 4). However, without requiring Lipschitz condition of the set valued map it is very hard to look for solutions of control problem, since in this case, the value function is not necessary lower semicontinuous (l.s.c.) and hence we can't neither characterize it as a l.s.c. solution of Hamilton-Jacobi-Bellman equation (HJB), nor use this last equation to provide sufficient conditions of optimality. To overcome this situation, another approach by means of duality can be used to study control problem (see [29-31]). Unfortunately, without Lipschitz and convex assumptions, duality gaps may occur. Our attention in this paper is focused upon 
duality. The duality combined with HJB equation as it presented here, contains two features: first we establish a weak duality between control problem (primal problem) and a dual problem (called intermediary dual problem) without Lipschitz assumption, and then, thanks to additional assumptions involving the relationship between monotonicity properties and the proximal subdifferential, we give a more precise estimation of the cost function of control problem. Another feature is the fact that under additional Lipshitz assumption, we show that proximal HJB equation combined with the estimation of the cost function confirm the optimality of a suspected candidate. Furthermore, in this case, we establish a strong duality between this optimal control problem and a dual problem involving upper hull of l.s.c. proximal supersolutions of the HJB equation (also with contingents supersolutions), which extend partially the duality established in [29] between an optimal control problem and a dual problem involving the continuous viscosity solutions of HJB equation. This strong duality provides necessary and sufficient conditions of optimality. We arrange the paper as follows: in Section 2, we state the control problem formulation and assumptions under which we work. In Section 3, we introduce the intermediary dual problem and prove weak duality and estimations. In Section 4, we prove sufficient conditions of optimality by combining estimation and the proximal HJB equation supersolutions. Thereafter, we introduce the proximal dual problem and prove strong duality. Finally, in Section 5, we provide necessary and sufficient conditions of optimality.

\section{PRIMAl PROBlem}

In this work, we are interested by the following Mayer optimal control problem dealing with differential inclusion:

$$
\left\{\begin{array}{l}
\alpha=\inf _{x} J(x):=g(x(T)), \\
\dot{x}(t) \in F(t, x(t)), \text { p.p. } t \in[0, T], \\
x(0)=x_{0} .
\end{array}\right.
$$

The infimum is taken over $x:[0, T] \rightarrow \mathbb{R}^{n}$ absolutely continuous $(x \in A C)$, a class of functions we call arcs.

The function $g: \mathbb{R}^{n} \rightarrow \mathbb{R}$, the set valued map $F:[0, T] \times \mathbb{R}^{n} \rightsquigarrow \mathbb{R}^{n}$ and $x_{0}$ are the datas of the problem. We recall that the function $x \in A C$ is a trajectory of $F$ if $\dot{x}(t) \in F(t, x(t))$ a.e. $t \in[0, T]$.

Consider the following set

$$
S_{[t, T]}(\xi):=\{x \text { trajectory of } F \text { on }[t, T] \text { such that } x(t)=\xi\} .
$$

Then an $\operatorname{arc} x$ is admissible for $(\mathrm{P})$ iff $x \in S_{[0, T]}\left(x_{0}\right)$.

The value function $V:[0,1] \times \mathbb{R}^{n} \rightarrow \mathbb{R} \cup\{ \pm \infty\}$ associated with the problem (P) is given as follows:

$$
V(t, \xi)=\inf \left\{g(x(T)): x \in S_{[t, T]}(\xi)\right\}
$$




\section{Remark 1.1.}

- Sometimes we deal with constraints as $x(T) \in K$ (see [21] and [22]), these type of constrains may be penalized by taking

$$
G(.):=g(.)+\Psi_{K}(.),
$$

where $\Psi_{K}($.$) is the indicator function$

$$
\Psi_{K}(x)=\left\{\begin{array}{l}
0 \text { if } x \in K \\
+\infty \text { otherwise }
\end{array}\right.
$$

- It is easy to show that $V$ satisfy the following assertions:

(1)

$$
V(T, \xi)=g(\xi), \quad \forall \xi \in \mathbb{R}^{n} .
$$

(2) For all $x \in S_{[t, T]}(\xi)$ the function

$$
s \rightarrow V(s, x(s)),
$$

is nondecreasing.

(3) If $\widehat{x}$ is an optimal solution of (P) then, for all $t \in[0, T]$,

$$
V(t, \widehat{x}(t))=g(\widehat{x}(T)) .
$$

\section{Basic hypotheses}

$H_{1}-g$ is lower semicontinuous (l.s.c.).

$H_{2}-\forall(t, x) \in[0, T] \times \mathbb{R}^{n}$, the set $F(t, x)$ is nonempty, compact, convex and there exist a constants $\gamma>0$ and $c>0$ such that, for all $(t, x)$

$$
v \in F(t, x) \Longrightarrow\|v\| \leq \gamma\|x\|+c
$$

where $\|$.$\| denotes the Euclidean norm.$

$H_{3}-F$ is upper semicontinuous, that is, for all $\varepsilon>0$, there exist $\delta>0$ such that, $\forall\left(t_{1}-t_{2}, x_{1}-x_{2}\right) \in B_{\delta}(0)$,

$$
F\left(t_{1}, x_{1}\right) \subset F\left(t_{2}, x_{2}\right)+\varepsilon B_{1}(0),
$$

where $B_{\delta}(0)$ denotes the open ball in $\mathbb{R}^{n+1}$, of center 0 and radius $\delta$ and $B_{1}(0)$ denotes the open ball in $\mathbb{R}^{n}$, of center 0 and radius 1 .

For some results, we require the local Lipschitz property of $F$ :

$H_{4}-F$ is locally Lipschitz, that is, for all $\varsigma>0$, there exist $c_{\varsigma}>0$ such that, $\forall\left(t_{1}-t_{2}, x_{1}-x_{2}\right) \in B_{\varsigma}(0)$,

$$
F\left(t_{1}, x_{1}\right) \subset F\left(t_{2}, x_{2}\right)+c_{\varsigma}\left\|\left(t_{1}-t_{2}, x_{1}-x_{2}\right)\right\| B_{1}(0) .
$$

The assumptions $H_{1}-H_{3}$ guarantee the existence of a solution of optimal control problem (P) (see [30] and also [12]). 
Proposition 1.2. Under the required assumptions $H_{1}-H_{3}$, the infimum of problem $(P)$ is achieved.

Furthermore, if the hypothesis $H_{4}$ is satisfied then the value function $V$ is lower semicontinuous.

\section{Estimation of the minimal value $\alpha$ of $(\mathrm{P})$}

\subsection{INTERMEDIARY DUAL PROBLEM}

Under the hypotheses $H_{1}-H_{3}$ and without Lipschitz assumption of the set value map $F$, the value function $V$ is not necessary l.s.c. and we can't then use HJB equation to characterize optimality. In this case, it will be interesting to look for an estimation of the minimal value, $\alpha$, of the problem $(\mathrm{P})$. The idea we follows is to establish a weak duality between the problem $(\mathrm{P})$ and an appropriate dual problem (called Intermediary dual problem) involving l.s.c. functions, and hence, thanks an adequate admissible function of the dual problem fulfilling some monotonicity properties, we provide a 'more precise' estimation of $\alpha$.

Let us first define the intermediary dual problem formulating as follows:

$$
\left(D_{I}\right) \quad\left\{\begin{array}{l}
\left.\left.\beta_{I}=\sup _{\varphi}\left\{T . \inf _{(t, \xi) \in] 0, T] \times \mathbb{R}^{n}} \frac{\varphi(t, \xi)-\varphi\left(0, x_{0}\right)}{t}+\varphi\left(0, x_{0}\right)\right\}\right] 0, T\right] \times \mathbb{R}^{n} \\
\varphi(T, .) \leq g(.) \text { on } \mathbb{R}^{n}
\end{array}\right.
$$

where $\varphi:[0, T] \times \mathbb{R}^{n} \rightarrow \mathbb{R} \cup\{+\infty\}$ is a l.s.c. function.

We now study the relationship between the primal problem $(\mathrm{P})$ and $\left(D_{I}\right)$. Without Lipschitz assumption of the multifunction $F$, we prove a weak duality result.

Proposition 2.1 (weak duality). Under the assumptions $H_{1}-H_{3}$, we have the following weak duality

$$
\alpha \geq \beta_{I}
$$

Proof. The assumptions $H_{1}-H_{3}$ guarantee that there exist a solution of the differential inclusion $\dot{x}=F(t, x(t)), x\left(t_{0}\right)=\xi$ for all $\left(t_{0}, \xi\right) \in[0, T] \times \mathbb{R}^{n}$. This means that there exist an admissible arc of the problem $(\mathrm{P})$. On the other hand, we have

$$
g(x(T)) \geq \varphi(T, x(T)),
$$

for each admissible $\operatorname{arc} x$ of $(\mathrm{P})$ and for each admissible function $\varphi$ of $\left(D_{I}\right)$.

By adding and substituting the term $\varphi\left(0, x_{0}\right)$, we obtain

$$
g(x(T)) \geq \varphi(T, x(T))-\varphi\left(0, x_{0}\right)+\varphi\left(0, x_{0}\right)
$$

which implies that

$$
g(x(T)) \geq T . \frac{\varphi(T, x(T))-\varphi\left(0, x_{0}\right)}{T}+\varphi\left(0, x_{0}\right) .
$$


So,

$$
g(x(T)) \geq T . \inf _{(t, x) \in] 0, T] \times \mathbb{R}^{n}} \frac{\varphi(t, x)-\varphi\left(0, x_{0}\right)}{t}+\varphi\left(0, x_{0}\right) .
$$

This occur for all $x$ and $\varphi$ admissible for $(\mathrm{P})$ and $\left(D_{I}\right)$ respectively.

It follows that

$$
\min _{x} g(x(T)) \geq \sup _{\varphi}\left\{T . \inf _{(t, x) \in] 0, T] \times \mathbb{R}^{n}} \frac{\varphi(t, x)-\varphi\left(0, x_{0}\right)}{t}+\varphi\left(0, x_{0}\right)\right\},
$$

and hence

$$
\alpha \geq \beta_{I}
$$

The weak duality proved in Proposition 2.1 may be shown as a first estimation of $\alpha$.

\subsection{Estimation of the minimal value, $\alpha$, of $(\mathrm{P})$}

We have established a weak duality, but in the framework of hypotheses $H_{1}-H_{3}$, the duality gaps may occur and strong duality may fails to exist. Our aim here is to invoke the concept of proximal subdifferential, since it present a suitable properties of monotonicity, to provide a more precise estimation of the minimal value, $\alpha$, of $(\mathrm{P})$. As it is well known in literature, we define a proximal subdifferential as follows (for more details, see $[12,15,17]$ ).

Definition 2.1. Let $\varphi:[0, T] \times \mathbb{R}^{n} \rightarrow(-\infty,+\infty]$ be an extended-valued lower semicontinuous function. The proximal subdifferential of $\varphi$ at $(t, x) \in[0, T] \times \mathbb{R}^{n}$ is given by:

$\partial_{p} \varphi(t, x):=\left\{\xi \in \mathbb{R}^{n+1}: \exists \sigma>0, \delta>0\right.$ s.t. $\varphi(s, y) \geq \varphi(t, x)-\sigma \|(t, x)-$ $\left.(s, y) \|^{2}+\langle\xi,(t, x)-(s, y)\rangle \quad \forall(s, y) \in(t, x)+\delta B\right\}$,

where $\langle a, b\rangle$ denotes the inner product of the vectors $a$ and $b$ and $B$ denotes the open unit ball.

Theorem 2.2. Under the assumptions $H_{1}-H_{3}$.

Let $\widehat{x} \in S_{[0, T]}\left(x_{0}\right), \widehat{\varphi}$ admissible for $\left(D_{I}\right)$ and consider the following function $W: t \in[0, T] \longrightarrow \widehat{\varphi}(t, \widehat{x}(t))$ such that

(1) For each $t \in[0, T]$ fixed, if $\xi \in \partial_{p} W(t)$ then $\xi \leq 0$;

(2) $\widehat{\varphi}(T, \widehat{x}(T))=g(\widehat{x}(T))$.

Then we have the following estimation of $\alpha$ :

$$
g(\widehat{x}(T))+T \inf _{(t, x) \in] 0, T] \times \mathbb{R}^{n}} \frac{\widehat{\varphi}(t, x)-\widehat{\varphi}\left(0, x_{0}\right)}{t} \leq \alpha \leq g(\widehat{x}(T)) .
$$


Proof. Condition (2) implies that $g(\widehat{x}(T))=\widehat{\varphi}(T, \widehat{x}(T))$ and condition (1) imply that the function $W$ is decreasing.

In particular,

$$
W(T) \leq W(0)
$$

Which means that

$$
\widehat{\varphi}(T, \widehat{x}(T)) \leq \widehat{\varphi}\left(0, x_{0}\right)
$$

Hence,

$$
g(\widehat{x}(T)) \leq \widehat{\varphi}\left(0, x_{0}\right)
$$

On the other hand, for all $x$ admissible for $(\mathrm{P})$, we have

$$
g(x(T))-\widehat{\varphi}(T, x(T)) \geq 0 .
$$

So, by combining the last two inequalities, we obtain for all $x$ admissible of $(\mathrm{P})$, that

$$
g(\widehat{x}(T)) \leq \widehat{\varphi}\left(0, x_{0}\right)+g(x(T))-\widehat{\varphi}(T, x(T)) .
$$

It follows that

$$
g(\widehat{x}(T)) \leq g(x(T))-T \cdot \frac{\widehat{\varphi}(T, x(T))-\widehat{\varphi}\left(0, x_{0}\right)}{T},
$$

and hence for all $x$ admissible for $(P)$

$$
g(\widehat{x}(T)) \leq g(x(T))-T . \inf _{(t, x) \in] 0, T] \times \mathbb{R}^{n}} \frac{\widehat{\varphi}(t, x)-\widehat{\varphi}\left(0, x_{0}\right)}{t} .
$$

By taking the infimum of $g(x(T))$ over all admissible $\operatorname{arcs} x$, we obtain that

$$
g(\widehat{x}(T))+T . \inf _{(t, x) \in] 0, T] \times \mathbb{R}^{n}} \frac{\widehat{\varphi}(t, x)-\widehat{\varphi}\left(0, x_{0}\right)}{t} \leq \alpha \leq g(\widehat{x}(T)),
$$

as required.

Remark 2.3. By introducing the Dini subderivative (also called contingents subderivative) of a l.s.c. function $\phi:[0, T] \times \mathbb{R}^{n} \rightarrow \mathbb{R} \cup\{+\infty\}$ defined by

$$
D \phi(t, x)(s, v)=\liminf _{s^{\prime} \rightarrow s ; v^{\prime} \rightarrow v, h \rightarrow 0^{+}} \frac{\phi\left(t+h s^{\prime}, x+h v^{\prime}\right)-\phi(t, x)}{h} .
$$

We can substitute the condition (1) of Theorem 2.2 by the following condition: $1^{\prime}-\forall(t, x) \in \operatorname{Dom}(\widehat{\varphi}): t<T \quad \inf _{v \in F(t, x)} D \widehat{\varphi}(t, x)(1, v) \leq 0$,

where $\widehat{\varphi}$ is the l.s.c. function of Theorem 2.2 and $\operatorname{Dom}(\widehat{\varphi}):=\{(t, x) \in[0, T] \times$ $\left.\mathbb{R}^{n} / \hat{\varphi}(t, x)<+\infty\right\}$. 
In fact, under assumptions $H_{2}$ and $H_{3}$, this condition is equivalent, (see [22]), to following:

For all $(t, x) \in[0, T] \times \mathbb{R}^{n}$, there exist $\bar{x} \in S_{[t, T]}(x)$, such that $\forall s \in[t, T]$,

$$
\widehat{\varphi}(s, \bar{x}(s)) \leq \widehat{\varphi}(t, x)
$$

In other words, the function, $\widehat{\varphi}(., \bar{x}()):. t \in[0, T] \longrightarrow \widehat{\varphi}(t, \bar{x}(t))$, is decreasing. So, the estimation (2.1) is true for $\operatorname{arc} \bar{x}$.

\section{Proximal dual problem}

This section is devoted to study the Mayer problem $(\mathrm{P})$ in the case where the additional assumption $H_{4}$ is fulfilled. Let us first recall the concept of proximal supersolution of the HJB equation.

Consider the lower Hamiltonian given by

$$
h:(t, x, \xi) \in[0, T] \times \mathbb{R}^{n} \times \mathbb{R}^{n} \rightarrow \min _{v \in F(t, x)}\langle v, \xi\rangle
$$

where $F$ is the set valued map of problem $(\mathrm{P})$ and $\langle a, b\rangle$ denotes the inner product of the vectors $a$ and $b$ and define the augmented Hamiltonian

$$
\bar{h}:(t, x, \theta, \xi) \in[0, T] \times \mathbb{R}^{n} \times \mathbb{R} \times \mathbb{R}^{n} \rightarrow \theta+h(t, x, \xi)
$$

Definition 3.1. Let $\varphi:[0, T] \times \mathbb{R}^{n} \rightarrow \mathbb{R} \cup\{+\infty\}$ a l.s.c. function. We say that the function $\varphi$ is a l.s.c. proximal supersolution of HJB equation iff

$$
\bar{h}\left(t, x, \partial_{p} \varphi(t, x)\right) \geq 0, \quad \forall(t, x) \in[0, T] \times \mathbb{R}^{n} .
$$

Remark 3.1. Equation (3.1) should be understood in the following sense:

$$
\bar{h}(t, x, \theta, \xi) \geq 0, \quad \forall(t, x) \in[0, T] \times \mathbb{R}^{n} \text { and } \forall(\theta, \xi) \in \partial_{p} \varphi(t, x) .
$$

Our purpose is to give solutions to a two following situations: first, can the estimation (2.1) provides sufficient conditions of optimality, when the functions involved in Problem $\left(D_{I}\right)$ are required to be proximal supersolutions of the HJB equation? On the other hand, the much of hamilton-Jacobi theory has revolved around the formulation of the appropriate final (initial or boundary) conditions under which we can characterize the value function as a unique solution of the HJB equation. It was shown that under final condition of the form $\liminf _{t \uparrow T, y \rightarrow x} \varphi(t, y)=g(x)=\varphi(T, x)$, for all $x \in \mathbb{R}^{n}$, the value function is the unique solution of the proximal (or contingent) HJB equation, see $[14,22,23]$. However, with a more general condition of the form

$$
\varphi(T, .) \leq g(.) \text { on } \mathbb{R}^{n}
$$


the unicity of solution of the HJB equation fail to hold. Indeed, if $\varphi:[0, T] \times$ $\mathbb{R}^{n} \rightarrow \mathbb{R} \cup\{+\infty\}$ is a l.s.c. supersolution of the HJB equation with the final condition (3.2), then each l.s.c. function $\phi:[0, T] \times \mathbb{R}^{n} \rightarrow \mathbb{R} \cup\{+\infty\}$ given by

$$
\phi(t, .)=\left\{\begin{array}{l}
\varphi(t, .)+c \text { for all } t \in[0, T[, \\
\varphi(T, .) \text { for } t=T,
\end{array}\right.
$$

where $c$ is an arbitrary constant, is another supersolution of the this equation. So, our second object is to characterize in such situations, by means of duality, the value function as a upper hull of proximal supersolutions of the HJB equation.

1st claim. Sufficient conditions

Proposition 3.2. Under the assumptions of Theorem 2.2. If, furthermore the assumption $\mathrm{H}_{4}$ is fulfilled and $\widehat{\varphi}$ is a proximal supersolution of HJB equation (3.1), then $\widehat{x}$ is optimal.

Proof. It is well known that $\widehat{\varphi}$ is proximal supersolution of HJB equation (3.1), iff $(\widehat{\varphi}, F)$ is strongly nondecreasing, (see [15] or Prop. 4.7, Chap. 1 in [30]), this means that, the function

$$
\widehat{\varphi}(., x(.)): t \in[0, T] \longmapsto \widehat{\varphi}(t, x(t)),
$$

is nondecreasing for each trajectory $x$ of $F$.

In the other words,

$$
\left.\left.\widehat{\varphi}(t, x) \geq \widehat{\varphi}\left(0, x_{0}\right) \forall(t, x) \in\right] 0, T\right] \times \mathbb{R}^{n} .
$$

This implies that

$$
\left.\left.\frac{\widehat{\varphi}(t, x)-\widehat{\varphi}\left(0, x_{0}\right)}{t} \geq 0 \quad \forall(t, x) \in\right] 0, T\right] \times \mathbb{R}^{n}
$$

and hence

$$
\inf _{(t, x) \in] 0, T] \times \mathbb{R}^{n}} \frac{\widehat{\varphi}(t, x)-\widehat{\varphi}\left(0, x_{0}\right)}{t} \geq 0 .
$$

This fact combined with the estimation (2.1) imply that

$$
g(\widehat{x}(T)) \leq g(\widehat{x}(T))+T . \inf _{(t, x) \in] 0, T] \times \mathbb{R}^{n}} \frac{\widehat{\varphi}(t, x)-\widehat{\varphi}\left(0, x_{0}\right)}{t} \leq \alpha \leq g(\widehat{x}(T)) .
$$

Hence, $\widehat{x}$ is an optimal solution of $(\mathrm{P})$.

Remark 3.3. In Proposition 3.2 we invoke hypotheses of Theorem 2.2, which imply that the function $t \mapsto W(t)=\widehat{\varphi}(t, \widehat{x}(t))$ is decreasing on $[0, T]$. On the other hand, the fact that $\widehat{\varphi}$ is also required to be a proximal supersolution of the HJB equation, implies that the function $t \mapsto \widehat{\varphi}(t, x(t))$ is nondecreasing on $[0, T]$, for all admissible arcs of $(\mathrm{P})$. We deduce that $t \mapsto \widehat{\varphi}(t, \widehat{x}(t))$ is constant on 
$[0, T]$, i.e., the function test $\widehat{\varphi}$ is constant along an optimal trajectory. This result keeping with the property (1.3) in Remark 1.1 saying that the value function $V$ is constant along an optimal trajectory.

2nd Claim. Strong duality

Consider the following dual problem (D):

$$
(D) \quad\left\{\begin{array}{l}
\beta=\sup _{\varphi} \varphi\left(0, x_{0}\right), \\
\text { The supremum is taken over } \varphi \text { satisfying, } \\
\bar{h}\left(t, x, \partial_{p} \varphi(t, x)\right) \geq 0 \forall(t, x) \in[0, T] \times \mathbb{R}^{n}, \\
\varphi(T, .) \leq g(.) \text { on } \mathbb{R}^{n} .
\end{array}\right.
$$

Theorem 3.4. Under the assumptions $H_{1}, H_{2}$ and $H_{4}$, we have

$$
\alpha=\beta \text {. }
$$

Proof. Theorem 2.1 asserts that $\alpha \geq \beta_{I}$, which implies that

$$
g(x(T)) \geq T . \inf _{(t, x) \in] 0, T] \times \mathbb{R}^{n}} \frac{\varphi(t, x)-\varphi\left(0, x_{0}\right)}{t}+\varphi\left(0, x_{0}\right) .
$$

This inequality is fulfilled for each admissible arc $x$ for $(\mathrm{P})$ and $\varphi$ satisfying $\varphi(T,.) \leq g($.$) on \mathbb{R}^{n}$, in particular for $\varphi$ which is proximal supersolution of the HJB equation.

On the other hand, as shown in the previous proposition, the fact that $\varphi$ is proximal solution of HJB equation implies that $\varphi$ satisfies the following inequality

$$
\left.\left.\frac{\varphi(t, x)-\varphi\left(0, x_{0}\right)}{t} \geq 0, \quad \forall(t, x) \in\right] 0, T\right] \times \mathbb{R}^{n} .
$$

So, taking into account of the inequality (3.3), we obtain that

$$
g(x(T)) \geq \varphi\left(0, x_{0}\right)
$$

for all $x$ and $\varphi$ satisfying $\bar{h}\left(t, x, \partial_{p} \varphi(t, x)\right) \geq 0$ and $\varphi(T,.) \leq g($.$) on \mathbb{R}^{n}$.

Hence

$$
\alpha \geq \beta .
$$

Conversely, we know from a result of Clarke, et al. [14], that the l.s.c. value function fulfills proximal HJB equation.

Hence, since our value function $V$, under the additional assumption $H_{4}$, is l.s.c., then it satisfies proximal HJB equation. So, we obtain

$$
V\left(0, x_{0}\right)=\alpha \geq \beta \geq V\left(0, x_{0}\right) .
$$

It follows that

$$
\alpha=\beta=V\left(0, x_{0}\right) .
$$


Remark 3.5. In the same context of Remark 2.3, By invoking the concept of Dini subderivative, Theorem 3.4 remains true when we substitute the fact that the functions involved in the dual problem (D) are a proximal supersolutions of the HJB equation by the following condition:

The function $\varphi$ satisfies

$$
\forall(t, x) \in \operatorname{Dom}(\varphi) \text { with } t>0 \quad \sup _{v \in F(t, x)} D \varphi(t, x)(-1,-v) \leq 0 .
$$

Under the hypotheses $H_{2}$ and $H_{4}$ this condition is equivalent to say, (see [22]), that for all $(t, x) \in[0, T] \times \mathbb{R}^{n}$, for all $z \in S_{[t, T]}(x)$, for all $s \in[t, T]$,

$$
\varphi(t, x) \leq \varphi(s, z(s))
$$

This implies that the function

$$
\varphi(., z(.)): t \in[0, T] \mapsto \varphi(t, z(t))
$$

is nondecreasing.

This fact permit us to establish, by using the same arguments of the proof of Theorem 3.4, that

$$
\alpha \geq \beta^{\prime}
$$

where $\beta^{\prime}$ is the supremum of the problem $(D)$ involving the functions $\varphi$ satisfying the inequality (3.4).

Conversely, in [22], the author prove that the l.s.c. value function satisfies the condition (3.4). It follows that

$$
V\left(0, x_{0}\right)=\alpha \geq \beta^{\prime} \geq V\left(0, x_{0}\right)
$$

Which implies that

$$
\alpha=\beta^{\prime}
$$

\section{NECESSARY AND SUFFICIENT CONDITIONS}

We now show that when the uniqueness result of the solution of the HJB equation not arise. We can use the strong duality between control problem and a dual problem involving upper hull of proximal supersolutions of HJB equation to provide necessary and sufficient conditions of optimality.

Theorem 4.1. Under assumptions of Theorem 3.4.

Let $\hat{x}$ be an admissible arc for $(P)$, then $\hat{x}$ is an optimal solution of $(P)$ iff there exist a sequence of l.s.c. functions $\left(\varphi_{n}\right)_{n}$ proximal supersolutions of HJB equation on $[0, T] \times \mathbb{R}^{n}$ and satisfying

$$
\varphi_{n}(T, .) \leq g(.) \text { on } \mathbb{R}^{n}
$$


and

$$
\lim _{n \rightarrow+\infty} \varphi_{n}\left(0, x_{0}\right)=J(\hat{x}) .
$$

Where $J$ is the cost function of the Mayer optimal control problem $(P)$.

Proof. Necessary conditions: assume that $\hat{x}$ is optimal for $(\mathrm{P})$.

Let $\left(\varphi_{n}\right)_{n}$ be a maximizing sequence for (D), it follows that

$$
\varphi_{n}(T, .) \leq g(.) \text { on } \mathbb{R}^{n}
$$

and that

$$
\lim _{n \rightarrow+\infty} \varphi_{n}\left(0, x_{0}\right)=\sup \varphi\left(0, x_{0}\right) .
$$

The supremum is taken over all $\varphi$ admissible for (D).

The equality (4.2) combined with duality Theorem 3.4 and the fact that $\alpha=$ $J(\hat{x})$ imply that

$$
\lim _{n \rightarrow+\infty} \varphi_{n}\left(0, x_{0}\right)=J(\hat{x}) .
$$

Sufficient conditions: assume now that there exist a sequence of l.s.c. functions $\left(\varphi_{n}\right)_{n}$ proximal supersolutions of HJB equation on $[0, T] \times \mathbb{R}^{n}$ and satisfying conditions (4.1) and (4.2).

Then for all $n, \varphi_{n}$ is admissible for (D) and hence

$$
\varphi_{n}\left(0, x_{0}\right) \leq \sup \varphi\left(0, x_{0}\right) .
$$

The supremum is taken over all $\varphi$ admissible for (D).

On the other hand, according to Theorem 3.4 and the fact that $\alpha \leq J(x)$ for each $x$ admissible for $(\mathrm{P})$, we conclude that

$$
\varphi_{n}\left(0, x_{0}\right) \leq J(x) .
$$

By letting $n$ tend towards 0 , we obtain that

$$
J(\hat{x}) \leq J(x) \text { for all } \operatorname{arcs} x \text { admissible for }(\mathrm{P})
$$

which implies that $\hat{x}$ is optimal for $(\mathrm{P})$ as required.

\section{Conclusion}

In this work, we have studied a differential inclusion optimal control problem in two cases. First, without requiring Lipschitz property of the set valued map, we have obtained estimations of minimal value of control problem by using duality and invoking monotonicity properties of proximal subdifferential. On the other hand, in the context of Lipschitz property, we have proved that proximal HJB equation combined with estimation of $\alpha$ confirm the optimality of a suspected candidate. Furthemore, we have established a strong duality between this optimal control problem and a dual problem involving l.s.c. proximal supersolutions of the HJB 
equation (respectively with contingent supersolutions). This strong duality provided necessary and sufficient conditions of optimality. This result, extend (and is in keeping with) the duality established in [29] between an optimal control problem and a dual problem involving the continuous viscosity solutions of HJB equation. Indeed, Clarke and Ledyeav [16] (see also [14]), proved that the three concepts of generalized solutions of HJB equation, namely, viscosity solution $[5,6,9,10,18,19]$, proximal solution [15] and Subbotin minmax solution are equivalents.

In our future work, we hope apply this proximal duality to optimal control problems with viability constraint on state $(x(t) \in K \quad \forall t \in[0, T])$.

\section{REFERENCES}

[1] J.P. Aubin, Viability theory. Birkhäuser Boston Inc. (1991).

[2] J.P. Aubin and A. Cellina, Differential inclusions. A Series of Comprehensive Studies in Mathematics, Springer-Verlag (1984).

[3] J.P. Aubin and I. Ekeland, Applied nonlinear analysis. A Wiley Interscience Publication, John Wiley \& Sons (1984).

[4] J.P. Aubin and H. Frankowska, Set valued analysis. Birkhäuser (1990).

[5] M. Bardi and I. Capuzzo-Dolcetta, Optimal control and viscosity solutions of HamiltonJacobi-Bellman equations. Birkhäuser (1997).

[6] G. Barles, Solutions de viscosité des équations de Hamilton-Jacobi. Mathématiques \& Applications, Springer-Verlag (1994).

[7] E.N. Barron, Viscosity solutions and analysis in $\mathrm{L}^{\infty}$, in Nonlinear analysis, differential equations and control, edited by F.H. Clarke and R. Stern. NATO Sciences Series (1999).

[8] E.N. Barron, L.C. Evans and R. Jensen, Viscosity solutions of Isaacs' equations and differential games with Lipshitz controls. J. Differ. Equ. 53 (1984) 213-233.

[9] E.N. Barron and R. Jensen, Semicontinuous viscosity solutions for Hamilton-Jacobi equations with convex Hamiltonians. Commun. Partial Differ. Equ. 15 (1990) 1713-1742.

[10] E.N. Barron and R. Jensen, Optimal control and semicontinuous viscosity solutions. Proc. Am. Math. Soc. 113 (1991) 397-402.

[11] C.W. Clark and J.M. Conrad, Natural resource economics. Cambridge University PressBirkhäuser (1995).

[12] F.H. Clarke, Optimization and nonsmooth analysis. Society for Industrial and Applied Mathematics, Philadelphia (1983).

[13] F.H. Clarke, Yu.S. Ledyaev and Stern R.J., Invariance, monotonicity, and applications. Nonlinear analysis, differential equations and control, edited by F.H. Clarke and R. Stern. NATO Sciences Series (1999).

[14] F.H. Clarke, Yu.S. Ledyaev and P.R. Wolenski, Qualitative properties of trajectories of control systems: a survey. J. Dyn. Control Syst. 1 (1995) 1-48.

[15] F.H. Clarke, Yu.S. Ledyaev, R.J. Stern and P.R. Wolenski, Nonsmooth analysis and control theory. Graduate Texts In Mathematics, Springer (1998).

[16] F.H. Clarke and Yu.S. Ledyaev, Mean value inequalities in Hilbert space. Trans. Am. Math. Soc. 344 (1994) 307-324.

[17] F.H. Clarke, Yu.S. Ledyaev and P.R. Wolenski, Proximal analysis and minimization principles. J. Math. Anal. Appl. 196 (1995) 722-735.

[18] M.G. Crandall and P.L. Lions, Viscosity solutions of Hamilton-Jacobi equations. Trans. Am. Math. Soc. 277 (1983).

[19] M.G. Crandall, L.C. Evan and P.L. Lions, Some properties of viscosity solutions of HamiltonJacobi equations. Trans. Am. Math. Soc. 282 (1984).

[20] L.C. Evan and M.R. James, The Hamilton-Jacobi-Bellman equation for time-optimal control. SIAM J. Control Optim. 27 (1989) 1477-1489. 
[21] H. Frankowska, Equations de Hamilton-Jacobi contingentes. C. R. Acad. Sci. Série I 304 (1987).

[22] H. Frankowska, Lower semicontinuous solutions of Hamilton-Jacobi-Bellman equations. SIAM J. Control Optim. 31 (1993) 257-272.

[23] H. Frankowska, S. Plaskacz and T. Rzeżuchowski, Measurable viability theorem and the Hamilton-Jacobi-Bellman equations. J. Diff. Equ. 116 (1995) 265-305.

[24] P.L. Lions and P.E. Souganidis, Differential Games, Optimal control and directional derivatives of viscosity solutions of Bellman's and Isaacs' equations. SIAM J. Control Optim. 23 (1985) 566-583.

[25] J.P. Penot and M. Volle, Explicit solutions to Hamilton-Jacobi equations under mild continuity and convexity assumptions. J. Nonlinear Convex Anal. 1 (2000) 177-199.

[26] N. Raïssi, Analyse proximale en optimisation. Ph.D. thesis, Université Montréal, Faculté des Arts et des Sciences, Canada (1987).

[27] T.R. Rockafellar, Existence theorems for general control problem of Bolza and Lagrange. Adv. Math. 15 (1975) 312-337.

[28] M. Serhani and N. Raïssi, Nonconvex duality and generalized solutions of HJB equation in optimal control problem, in Conférence Internationale en Optimisation FGI, 4-9 Septembre, Montpellier, France (2000).

[29] M. Serhani and N. Raïssi, Nonconvex duality and viscosity solutions of HJB equation in optimal control problem. J. Convex Anal. 9 (2002) 625-648.

[30] M. Serhani, Dualité et analyse non lisse : Optimisation non convexe, contrôle optimal et solutions généralisées de l'équation de HJB. Ph.D. thesis. University Ibn Tofail, Morocco (2002).

[31] R. Vinter, Convex duality and nonlinear optimal control. SIAM J. Control Optim. 31 (1993) $518-538$.

[32] R. Vinter and P.R. Wolenski, Hamilton-Jacobi theory for optimal control problems with data measurable in time. SIAM J. Control Optim. 28 (1990) 1404-1419.

[33] J.J. Ye, Discontinuous solutions of the Hamilton-Jacobi equations for exit time problems. SIAM J. Control Optim. 38 (2000) 1067-1085.

[34] L.C. Young, Lectures on the calculus of variations and optimal control theory. W.B. Saunders Company (1969). 